test 8 (MMT8) were collected. Data were reported as median \pm interquartile range; non-parametric tests were used for the analysis.

Results: Sixty-three patients presented muscular edema in at least one muscle. Patients with muscular edema had higher CK levels (1506 \pm 1976 vs $235 \pm 224 p<0.001)$ lower disease duration $(9.5 \pm 47.0$ vs $19.8 \pm 97.2$ $\mathrm{p}=0.031)$. and lower MMT8 $(63.6 \pm 11.2$ vs $69.8 \pm 10.7 \mathrm{p}=0.031)$.

Forty-eight patients presented fatty infiltrates that were more frequent in older patients $(63.1 \pm 11-3$ vs $52.8 \pm 13.7 \mathrm{p}=0.01)$ and in those with longer disease duration $(39.1 \pm 95.4$ vs $9.5 \pm 12.5 \mathrm{p}=0.024)$. CK levels and MMT8 were not different in patients with or without muscular fatty infiltrates. With the multivariate analysis the disease duration represents the only independent factor for the presence of muscular fatty infiltrates $(p=0.05)$. Muscular atrophy was present in 17 patients but it was not correlated to age and disease duration. CK and MMT8 were not different in presence/ absence of fatty infiltrates or muscular atrophy.

Edema and atrophy were not different between poly- and dermatomyositis. The fatty infiltrate was more present in the posterior compartment of the tights (biceps femoris and semitendinosus muscle) in patients with PM compared to DM $(68.2 \%$ vs $29.6 \% \mathrm{p}=0.046)$

Conclusion: The alterations identified with $\mathrm{MRI}$ in our cohort changed according to the disease duration and the age of the patients. In particular, fatty infiltration was more frequent in patients with longer disease duration, but was not associated to CK levels and muscular weakness. Moreover, fatty infiltration was prevalent in the posterior compartment of the tights in PM patients.

Muscular $\mathrm{MRI}$ is widely used in the diagnosis and follow-up but the clinical meanings of the different alterations still need to be investigated.

Disclosure of Interests: : Simone Barsotti: None declared, Barbara Mugellini: None declared, Alessandra Tripoli: None declared, Giacomo Aringhieri: None declared, Chiara Cardelli: None declared, Elisa Cioffi: None declared, Virna Zampa: None declared, Davide Caramella: None declared, Marta Mosca Paid instructor for: GlaxoSmithKline, Lilly, UCB, Rossella Neri: None declared

DOI: 10.1136/annrheumdis-2019-eular.6784

\section{SAT0261 CHARACTERISTICS ASSOCIATED TO SCLERODERMAL RENAL CRISIS, AND INCIDENCE VARIATION OVER TIME IN THE RESCLE REGISTRY}

$\underline{\text { Xavier Pla Salas }}^{1}$, Carles Tolosa ${ }^{2}$, Eduardo L. Callejas-Moraga ${ }^{2}$, Alfredo Guillén del Castillo ${ }^{3}$, Jose Antonio Todolí Parra ${ }^{4}$, Luis Trapiella Martínez ${ }^{5}$, Mónica Rodríguez Carballeira ${ }^{6}$, Adela Marín Ballvé ${ }^{7}$, Isabel Perales Fraile ${ }^{8}$ Vicent Fonollosa-Pla ${ }^{9}$, Carmen Pilar Simeón-Aznar ${ }^{10}$, On Behalf of Rescle Investigators, Autoimmune Diseases Study Group (Geas) ${ }^{11}$. ${ }^{1}$ Consorci Hospitalari de Vic, Unit of Systemic Autoimmune Diseases. Department of Internal Medicine., Vic, Spain; ${ }^{2}$ Corporació Sanitaria Universitaria Parc Taulí, Department of Internal Medicine, Sabadell, Spain; ${ }^{3}$ Hospital Vall d'Hebron, Unit of Autoimmune Diseases. Department of Internal Medicine., Barcelona, Spain; ${ }^{4}$ Hospital Universitario y Politécnico La Fe, Department of Internal Medicine, Valencia, Spain; ${ }^{5}$ Hospital de Cabueñes, Unit of systemic autoimmune diseases. Department of Internal Medicine., Gijón, Spain; ${ }^{6}$ Hospital Universitario Mútua de Terrassa., Department of Internal Medicine., Terrassa, Spain; ${ }^{7}$ Hospital Clínico Universitario Lozano Blesa., Unit of Autoimmune Diseases. Department of Internal Medicine., Zaragoza, Spain; ${ }^{8}$ Hospital Universitario Rey Juan Carlos, Department of Internal Medicine, Móstoles, Spain; ${ }^{9}$ Hospital Universitario Vall d'Hebron, Unit of Autoimmune Diseases. Department of Internal Medicine., Barcelona, Spain; ${ }^{10} \mathrm{Hospital}$ Universitario Vall d'Hebrón, Unit of Autoimmune Diseases. Department of Internal Medicine., Barcelona, Spain; ${ }^{11}$ Sociedad Española de Medicina Interna (SEMI), Madrid, Spain

Background: Scleroderma Renal Crisis (SRC) is a serious complication of Systemic Sclerosis (SSc). Nowadays, it seems that there is a reduction in its prevalence and mortality ${ }^{1}$

Objectives: To evaluate the characteristics of patients with SRC in a large cohort of SSc pacients. To investigate predictors of SRC, and epidemiologic differences over time.

Methods: 1933 patients were collected in ongoing registry of Spanish SSc pacients - RESCLE. We did descriptive study and epidemiologic analysis.

Results: Out of 1933 SSc, 43 (2.2\%) developed SRC. Univariate analysis showed significant differences of SRC vs. non-SRC cases: SSc subtypes: diffuse cutaneous SSc (dcSSc), $72 \%$ vs. 19\%; limited cutaneous SSc (IcSSc), $26 \%$ vs. 61\%. Demographics: Female gender, $77 \%$ vs. $89 \%$; time from SSc onset to SSc diagnosis, $3.0 \pm 8.0$ vs. $6.6 \pm 9.5$ years; arterial hypertension (HT), $56 \%$ vs. $32 \%$. 1st manifestation: Raynaud's phenomenon (RP), $68 \%$ vs. $82 \%$. Clinical manifestations: RP, $88 \%$ vs. $96 \%$; digital ulcers, $70 \%$ vs. $38 \%$; arthritis, $45 \%$ vs. $20 \%$; myositis, $30 \%$ vs. $13 \%$; joint contractures, $45 \%$ vs. $18 \%$; intestinal involvement, $24 \%$ vs $11 \%$; malabsorption, $24 \%$ vs. $7 \%$; interstitial lung disease $58 \%$ vs. $41 \%$ pulmonary HT, $56 \%$ vs. $29 \%$; pericardial effusion, $28 \%$ vs. $7.4 \%$; pericarditis, $24 \%$ vs. $8.3 \%$; ischemic cardiopathy, $31 \%$ vs. $12 \%$; diastolic dys function, $67 \%$ vs. $34 \%$. Capillaroscopy: active pattern $77 \%$ vs. $33 \%$ Immunological data: anti-Topoisomerase I, $39 \%$ vs. $20 \%$; anti-centromere $15 \%$ vs. $49 \%$; anti-RNApol III, $45 \%$ vs. $11 \%$. Prognosis: Overall mortality, $56 \%$ vs. $18 \%$; SSc-related mortality, $83 \%$ vs. $49 \%$. Survival at 5, 10, 20 and 30 years was $73 \%$ vs. $96 \%, 56 \%$ vs. $92 \%, 28 \%$ vs. $80 \%$, and $28 \%$ vs. $67 \%$, respectively. Treatment: ACEI use, $35 \%$ vs. $14 \%$, corticoid use, $51 \%$ vs. $25 \%$. Multivariate analysis: dsSSc subtype, RR 22.68 (5.81 88.51) $\mathrm{p}<0.001$; intestinal malabsorption, $R R 7.35 \quad(2.55-21.18) \quad \mathrm{p}<0.001$ and active capillaroscopy pattern RR 7.26 (1.61-32.7) $p<0.010$. Prevalence of SRC $(\mathrm{P})$ in dcSSc subtype $7.8 \%$, and in IcSSc subtype $0.9 \%$. P over decades: P-80's, 3.8\%; P-90's, 2.7\%; P-00's: $2.3 \%$ and P-10's: $0.9 \%$ achieving statistical significance in the last decade RR: $0.33(0.13-0.85)$ $\mathrm{p}=0.014$

Conclusion: In RESCLE cohort, SRC predominated in dcSSc patients, and it was associated to intestinal malabsorption, and an active pattern by capillaroscopy. SRC showed a very poor prognosis. Finally, we evidenced a decreasing prevalence of SRC over time in our cohort.

\section{REFERENCE}

[1] Turk M, et al. The Frequency of Scleroderma Renal Crisis over Time: A Metaanalysis. J Rheumatol 2016; 43: 1350.

Disclosure of Interests: None declared DOI: 10.1136/annrheumdis-2019-eular.3406

\section{SAT0262 MODIFIED ACR COMPOSITE RESPONSE INDEX IN SYSTEMIC SCLEROSIS SCORE SHOWS SENSITIVITY AND EXTERNAL VALIDATION TO MEASURE MAGNITUDE OF RESPONSE AT 12 MONTHS IN DIFFUSE CUTANEOUS SYSTEMIC SCLEROSIS}

Giuseppina Abignano $^{1,2,3}$, Antonio Carriero ${ }^{1,3}$, Sookhoe Eng ${ }^{1,2}$, Francesco Del Galdo ${ }^{1,2}$. $^{1}$ Leeds Institute of Rheumatic and Musculoskeletal Medicine, University of Leeds, Leeds, United Kingdom; ${ }^{2}$ NIHR Leeds Biomedical Research Centre, Leeds Teaching Hospitals NHS Trust, Leeds, United Kingdom; ${ }^{3}$ Rheumatology Institute of Lucania (IReL), Rheumatology Department of Lucania, Potenza, Italy

Background: The Composite Response Index in Systemic Sclerosis (CRISS) is a 2-step process for the probability of improvement of patients with diffuse cutaneous systemic sclerosis (dcSSc) ranging from 0.0 (no improvement) to 1.0 (1). The limitation of probability is that it does not measure magnitude of response and it cannot be negative, hence reflecting worsening.

Objectives: Here we aimed to test the performance of CRISS numerator normalised by baseline values to assess $\%$ change at 12 months in an observational cohort.

Methods: Consecutive dcSSc patients were included in the study at a single centre. Clinical data were collected at baseline and 12 months and CRISS calculated using the published formula. To explore the quantitative value of the numerator, each of the 5 domains was corrected for their baseline value (e.g (12 months baseline)/baseline $=$ Delta\%) while keeping their original relative weight in the formula. The weighted sum was then divided by the number of domains. The modified CRISS (mCRS) was then compared with the original CRISS score and with another composite score validated in randomized controlled trials, the Global Ranked Composite Score (GRCS) (2). Death and organ failure were also included and assigned the worst negative values. Spearman's test was used for Prism 7 correlation analysis. $P<0.05$ was considered significant.

Results: Thirty-three dcSSc patients were enrolled. Twenty-one patients had a CRISS $=0 \%$. Twelve patients had a positive CRISS ranging from $1 \%$ to $77 \%$. $18 / 21$ patients with CRISS $=0 \%$ showed a negative mCRS (negative response $=$ worsening) ranging from $-9 \%$ to $-2500 \%$. 10/12 with positive CRISS showed a corresponding positive mCRS ranging from $1 \%$ to $22 \%$. mCRS had a significant correlation with CRISS $(r=0.5, p=$ $0.003)$ and GRCS ( $r=0.7, p<0.0001)$ despite the low number of patients enrolled.

Conclusion: Normalization by baseline data within CRISS numerator offers a quantitative score to assess magnitude of response, considering the 5 domains and their relative weight within the original CRISS. The mCRS 
measures magnitude of response rather than probability of response and includes the possibility of measuring worsening of disease. Application of the simple formula to the results of RCTs adopting CRISS as explorative efficacy objective will determine the validity of mCRS in offering quantitative assessment of response in SSc.

\section{REFERENCE}

[1] Khanna D, Berrocal VJ, Giannini EH, et al. The American College of Rheumatology Provisional Composite Response Index for Clinical Trials in Early Diffuse Cutaneous Systemic Sclerosis. Arthritis Rheumatol 2016;68:299311.

[2] Sullivan KM, Goldmuntz EA, Keyes-Elstein L, et al. Myeloablative Autologous Stem-Cell Transplantation for Severe Scleroderma. N Engl J Med 2018; 378:35-47

Disclosure of Interests: None declared

DOI: 10.1136/annrheumdis-2019-eular.7962

\section{SAT0263 MALIGNANT CONDITIONS IN SYSTEMIC SCLEROSIS PATIENTS, SINGLE CENTER EXPIRIENCE}

Alexandra Balbir-Gurman ${ }^{1}$, Menahem Nahir $^{2}{ }^{2}$ Vika Shataylo ${ }^{3}$,Doron Markovits ${ }^{3}$, Yolanda Braun-Moscovici ${ }^{1} .{ }^{1}$ Rambam Health Care Campus, Rappaport Faculty of Medicine, Technion, Rheumatology Institute, Haifa, Israel; ${ }^{2}$ ambam Health Care Campus, Rappaport Faculty of Medicine, Technion, Rheumatology Institute, Haifa, Israel, ${ }^{3}$ Rambam Health Care Campus, Rheumatology Institute, Haifa, Israel

Background: Internal organ complications (interstitial lung disease [ILD], pulmonary hypertension [PAH], renal crisis [SRC], gastrointestinal involvement [GIT], etc.) have significant influence on scleroderma (SSc) patients' morbidity and mortality; malignancy could contribute to SSc outcome.

Objectives: We aim to assess the incidence of malignancy, clinical and laboratory factors associated with this complication.

Methods: We identified 43 patients with biopsy and/or imaging confirmed malignant conditions (SSc-C); additionally, case-control study was performed with identifying SSc patients without cancer (All-NC, N=86) divided into two subgroups: matching age (Age-NC, N-43) and matching disease duration (DD-NC, $\mathrm{N}=43$ ).

Results: Since 2004, 424 SSc patients were assessed at our center (340 EUSTAR registry participants); 43 (10.1\%) SSc-C patients (7 males [16.3\%]; age [years, standard deviation, SD] 68.1[12.7]; age at SSc onset 52.9[14.9]; age at $1^{\text {st }}$ cancer diagnosis 54.2[12.7]; smoking 12 [27.9] had various types of tumors: breast 9; lung 8; neck \& head 8 (brain-3, thyroid-3, parotis-1, laryngs-1); genito-urinary 8 (prostate-1, ovary-3, utery-4; GIT 6 (stomach 2, pancreas 2, colon 2); hematologic 3 (lymphoma 2, myeloma 1); skin 3; pheochromocytoma 1, carcinoid 2; sarcoma 1. Thirteen patients $(30.2 \%)$ developed cancer three years before or after SSc onset; 7 patients had cancer long before SSc; 6 patients had two cancers. Comparison between SSc-C patients and subgroups Age-NC, DD$\mathrm{NC}$ and All-NC patient's subgroup did not reveal any difference in term of disease subset, presence of antibodies to topoisomerase, centromere, RNA polymerase, and clinical features (digital ulcers, ILD, GIT \& heart involvement, $\mathrm{PAH}$, myositis, polyautoimmunity); there were no cases of SRC in SSC-C patients compared to $8(9.3 \%)$ patients in All-NC patients. Twenty-six (60.5\%) SSc-C patients died compared to 16 (37.2) in AgeNC $(P<0.08)$, DD-NC $(P<0.001)$, All-NC $(P<0.001)$. SSc related death was reported in $22.1 \%$ of All-NC patients and $16.3 \%$ of SSc-C ( $P=N S) ; 19$ patients dead from cancer $(73.1 \%)$. There were no differences between cancer and non-cancer patients in term of treatment with methotrexate, mycofenolate, azathioprine, cyclophosphamide, calcium blockers and iloprost; $24.3 \%$ SSc-C patients were treated with bosentan compared to $44.5 \%(P, 0.015)$.

Conclusion: Malignancy is an often and variable scleroderma complication; it could preclude SSc or appeared during SSc course. Patients with malignancy had higher mortality rate and mostly dead from cancer. There no cases of tumor in those patients who developed SRC. Patients treated with bosentan has less incidence of tumor. Prompt screening for cancer should be consider as a reasonable approach in caring SSc patients; early diagnosis and treatment of tumor may improve SSc patients' survival.

Disclosure of Interests: None declared

DOI: 10.1136/annrheumdis-2019-eular.8258

\section{SAT0264 \\ GAZE PATTERN ANALYSIS IN THE ASSESSMENT OF DIGITAL ULCERS IN PATIENTS WITH SYSTEMIC SCLEROSIS}

Thomas Moser ${ }^{1}$, Quentin Lohmeyer ${ }^{2}$,Mirko Meboldt ${ }^{2}$, Oliver Distler ${ }^{1}$,Mike O. Becker ${ }^{1} .{ }^{1}$ University Hospital Zürich, Rheumatology, Zürich, Switzerland; ${ }^{2}$ ETH Zürich, Mechanical and Process Engineering, Zürich, Switzerland

Background: The assessment of chronic wounds such as digital ulcers (DUs) in systemic sclerosis relies heavily on visual aspects. However, inter-rater variability in the assessment and definition of digital ulcers is low and there are no evidence-based data concerning the visual assessment of digital ulcers ${ }^{a}$.

Objectives: We analysed gaze pattern data in order to evaluate differences in the visual assessment of digital ulcers in systemic sclerosis patients.

Methods: We analysed gaze pattern data from 36 subjects: 9 expert medical professionals (EMP), 8 non-expert medical professionals (NEMP), 9 medical graduates (MG) and 10 lay persons (novices). Assessment was done using a mobile eye-tracking device that tracks eye movements of subjects. Twenty pictures from digital ulcers of SSc patients were presented to each subject, 30 seconds each and characteristics of gaze pattern data were analysed. The analysis comprised the scan path, the dwell times (for areas of interest, AOI), fixation counts and the entry time for each picture and subject. Areas of interest were defined as the wound ground, wound margin, wound surroundings and distracting and non-relevant parts of the pictures. The visual assessment of the digital ulcers was accompanied by questions for each subject about their assessment of the DUs, the expected healing of the ulcer, the medical treatment of the patient.

Results: Most significant differences were found between novices and medically educated groups (expert medical professionals, non-expert medical professionals, medical graduates - EMP, NEMP, MG). Dwell times in the wound area for novices differed statistically significant from all medically educated groups (1.76s-3.1s less). Above all, novices had lower dwell times in wound margin and wound surrounding and spent more time in areas with other distracting features of the picture and white space (up to 1.92s longer). Correspondingly, they had less fixation points and longer overall scan paths in wound areas. Similar gaze pattern data were observed for medically educated groups, however NEMP had a statistically significantly lower dwell time in the wound area than EMP. Questionnaire responses were compared to an expert opinion (gold standard) and EMP had significantly more correct answers on prognosis and treatment than MG for wound assessment, but not better than NEMP

However, treatment and prognosis were best in the EMP group with statistical difference to the NEMP and MG group.

Conclusion: For the first time, we provide evidence-based data on the visual assessment of digital ulcers in SSc. These data will be useful for the development of a structured educational programme for young physicians, rheumatology trainees and medical graduates. A key finding is that the visual assessment of digital ulcers should increasingly emphasize the importance of pathologic changes in the wound margin and surrounding area of the wound. Hence a structured approach should focus on all areas of the wound while discarding distracting visual information. An adequate terminology should be used alongside.

\section{REFERENCE}

[1] Hughes M et al., J Scleroderma Relat Disord. 2018 Jun 1;3(2):170-174.

Disclosure of Interests: : Thomas Moser: None declared, Quentin Lohmeyer: None declared, Mirko Meboldt: None declared, Oliver Distler Grant/research support from: Prof. Distler received research funding from Actelion, Bayer, Boehringer Ingelheim and Mitsubishi Tanabe to investigate potential treatments of scleroderma and its complications, Consultant for: Prof. Distler has/had consultancy relationship within the last 3 years with Actelion, AnaMar, Bayer, Boehringer Ingelheim, ChemomAb, espeRare foundation, Genentech/Roche, GSK, Inventiva, Italfarmaco, iQvia, Lilly medac, Medlmmune, Mitsubishi Tanabe Pharma, Pharmacyclics, Novartis, Pfizer, Sanofi, Serodapharm and UCB in the area of potential treatments of scleroderma and its complications. In addition, he had/has consultancy relationship within the last 3 years with $A$. Menarini, Amgen, Abbvie, GSK, Mepha, MSD, Pfizer and UCB in the field of arthritides and related disorders, Mike O. Becker: None declared DOI: 10.1136/annrheumdis-2019-eular 6800 